\title{
Percepción de riesgo y consumo de alcohol y tabaco en estudiantes de una preparatoria en Zacatecas
}

\author{
Delgadillo Legaspi Luz Ma., Cortaza Ramírez Leticia **, Calixto-Olalde Ma. Gloria***
}

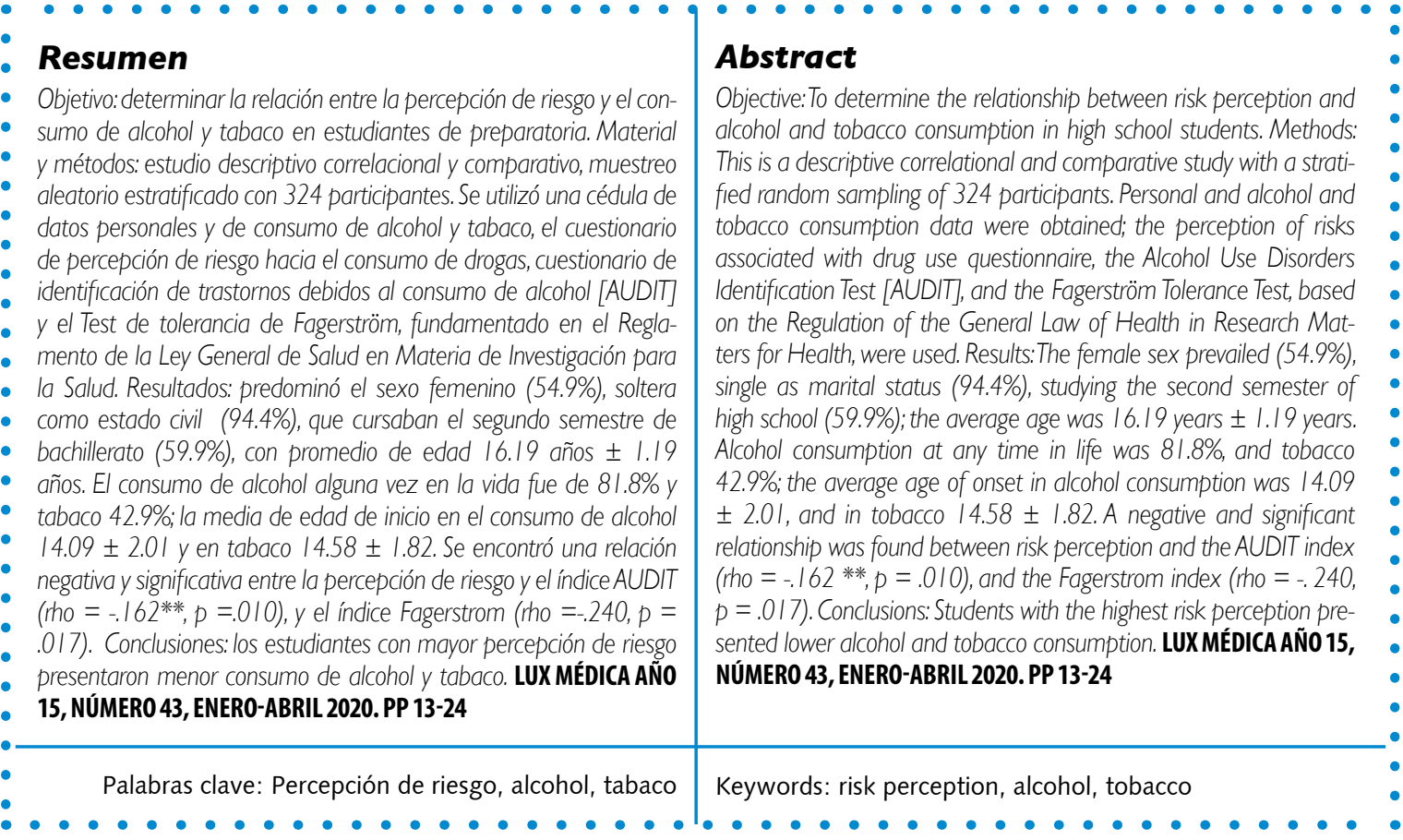

\section{Introducción}

La Organización Mundial de la Salud (OMS) considera al consumo de alcohol y tabaco como un problema de salud pública por la pesada carga social, económica y de salud que genera en todos los

\footnotetext{
Maestra en Ciencias de Enfermería, Estudiante de Doctorado en Ciencias de Enfermería, División de Ciencias de la Salud e Ingenierías, Campus Celaya-Salvatierra, Universidad de Guanajuato. Correo electrónico: Iuzmadeleg@gmail.com

** Dra. en Ciencias de Enfermería, Profesora de tiempo completo, Facultad de Enfermería, Universidad Veracruzana, Campus Minatitlán, Veracruz, correo electrónico: leticortaz@hotmail.com.

*** Dra. en Ciencias de Enfermería, Coordinadora del Programa de Doctorado en Ciencias de Enfermería, División de Ciencias de la Salud e Ingenierías, Campus Celaya Salvatierra, Universidad de Guanajuato, correo electrónico: gloriaco_26@hotmail.com.

Fecha de recibido: 8 de abril 2019

Fecha de aceptación: 8 de agosto 2019

Correspondencia: MCE. Luz Ma. Delgadillo Legaspi, Docente-investigador Unidad Académica de Enfermería, Universidad Autónoma de Zacatecas, Campus Universitario UAZ Siglo XXI, Carretera Zacatecas-Guadalajara, km 6, Ejido la Escondida, C.P. 98160, Teléfono: 9256690 , ext 4700 e-mail: luzmadeleg@gmail.com
} 
países. Debido al consumo de alcohol cada año mueren 3.3 millones de personas en el mundo, lo que representa el $5.9 \%$ del total de las defunciones. Considerado como un factor de riesgo para desarrollar trastornos mentales, enfermedades infecciosas como la tuberculosis, VIH/SIDA, enfermedades no trasmisibles cardiovasculares, cirrosis, algunos tipos de cáncer, suicidios y traumatismos derivados de la violencia, y los accidentes de tránsito; estos últimos especialmente afectan a personas jóvenes. Más de una cuarta parte $(27 \%)$ de los jóvenes de 15-19 años son bebedores; las mayores tasas de consumo de alcohol corresponden a Europa (44\%), las Américas (38\%) y el Pacífico Occidental (38\%)., ${ }^{1,2}$

El tabaquismo representa la primera causa de mortalidad prevenible en el mundo, es considerado como un problema prioritario de salud pública, por ser causa importante de enfermedad y discapacidad, se estima que entre 2010-2050 morirán 400 millones de personas a causa de enfermedades relacionadas con el tabaco. En América Latina, la carga de morbilidad atribuible al consumo de tabaco es muy elevada debido a la proporción de años de vida ajustados por discapacidad (AVAD) que se pierden cada año a consecuencia del consumo de tabaco. ${ }^{3-5}$ Algunos reportes mencionan que a nivel mundial existen más de 150 millones de adolescentes que consumen tabaco, con inicios a edades muy tempranas y con las consecuencias negativas como es la consolidación del hábito, prolonga el tiempo de inhalación directa de compuestos químicos del cigarro de los que se ha comprobado su toxicidad. (-7 $^{6-7}$

En México, la Encuesta Nacional de Adicciones (ENA) (2002 y 2011) y la Encuesta Nacional de Consumo de Drogas, Alcohol y Tabaco (ENCODAT) 2016 y 2017, reportan en la población adolescente de 12 a 17 años, un incremento en el uso alcohol sobre todo en el último mes, el cual pasó de $7.1 \%$ a $14.5 \%$ y $16.2 \%$, en hombres y en las mujeres de $2.7 \%$ a $11.6 \%$ y $15.4 \%$. La ingesta de alcohol diario pasó de $0.3 \%$ a $0.2 \%$ y $2.6 \%$; y en el patrón de consumo de alcohol consuetudinario de $1.8 \%$ a $1.0 \%$ y $4.1 \% .8,9$ En el caso de la ENA, el consumo de tabaco reportado en los adolescentes de $2011^{10}$ fue de $12.3 \%$ en el último año, la media de edad de inicio fue de 14.1 años, el consumo promedio fue de 4.1 cigarros por ocasión, $2.0 \%$ fuma diario, $10.4 \%$ lo hace en forma ocasional, y $8.1 \%$ presentó adicción a la nicotina. Por su parte, la ENCODAT 2016-201711 informa que 22.8\% fumó cigarros alguna vez en la vida, $7.8 \%$ lo hizo en el último año, y $4.9 \%$ en el último mes; de ellos $3.0 \%$ es mujer y $6.7 \%$ hombre; $0.5 \%$ fuma diariamente y $4.4 \%$ lo hace en forma ocasional.

Los datos del consumo de alcohol y tabaco en los adolescentes son preocupantes debido a que las cifras se mantienen o incrementan en las evidencias revisadas. Estos resultados son el reflejo de diversos factores, entre los que destacan la alta tolerancia del contexto social y la baja percepción de riesgo asociada al consumo de estas sustancias, con una serie de consecuencias a la salud de los adolescentes. ${ }^{12}$ 
Es importante tomar en cuenta que el consumo de alcohol y tabaco se asocia con algunas características de la adolescencia, debido a que se le considera como una etapa crítica en el desarrollo humano, por la multitud de cambios que se producen a nivel físico, psicológico y social, a la vez que representa el paso de la infancia a la adultez y se caracteriza por la búsqueda de la identidad personal del individuo, la autonomía e independencia, la adopción de roles de adulto, la concepción de inmunidad frente a los riesgos, que pone a prueba límites, vínculos y potencialidades para adaptarse a diversas circunstancias y favorece la búsqueda de sensaciones novedosas momentáneas e inmediatas. ${ }^{12-15}$

Además del efecto que estas sustancias producen en la salud, debido a que en este grupo etario puede producir déficit cognoscitivo y provocar daño cerebral, afecta la toma de decisiones lo que se asocia a otros problemas al adoptar conductas de riesgo como el inicio de la vida sexual activa sin protección con la consecuente presencia de infecciones de transmisión sexual, embarazos precoces, accidentes de tráfico, la adopción de estilos de vida poco saludables, el inicio en el consumo de sustancias adictivas a edades mucho más tempranas, en particular alcohol, tabaco y otras drogas. ${ }^{12-15}$

El consumo de alcohol y tabaco no es un problema que se presenta de un día para otro, esta conducta está íntimamente relacionada con diversos factores de riesgo considerados como todas aquellas situaciones en las cuales es más probable que se presente una conducta, ${ }^{12}$ entre ellos destacan el entorno social, la alta tolerancia del contexto hacia el consumo de estas sustancias, el bajo nivel de información sobre el alcohol y tabaco, baja autoestima, actitudes antisociales, insatisfacción con el uso del tiempo libre, la exposición a la oportunidad y la tentación al consumo, algunos eventos estresantes de la vida, carencia de habilidades para la toma de decisiones. Adicionalmente, tener actitudes y conductas fundamentadas de que el consumo de alcohol y tabaco no tiene riesgos y que favorece la diversión; es decir, que exista una baja percepción de riesgo hacia el consumo de estas sustancias. ${ }^{12,16,17}$

El estudio de la percepción de riesgo ha sido considerada como una de las variables que presentan mayor impacto en el inicio y mantenimiento del consumo de sustancias en los jóvenes, y es considerado como un factor importante para prevenir las adicciones. ${ }^{16,18}$ La percepción de riesgo se define como aquellos juicios o intuiciones que se tienen sobre la peligrosidad de una situación, y se le considera como el conocimiento sobre los daños y las consecuencias del consumo de drogas y la severidad atribuida a éstas. Cuando los adolescentes cuentan con alta percepción de riesgo, tienen menor probabilidad de consumir alcohol y tabaco frente a los que tienen una baja percepción de riesgo, debido a que la percepción del riesgo puede funcionar como reguladora de la motivación para que se presente la conducta. ${ }^{16-18}$ 
Son pocos los estudios encontrados que relacionan la variable de percepción de riesgo y el consumo de alcohol en adolescentes, las investigaciones localizadas reportan predominio del nivel medio de percepción de riesgo en ambos sexos, ${ }^{18}$ quienes no consumen alcohol presentaron mayor percepción de riesgo comparado con los consumidores. ${ }^{17-19}$ En la asociación de la percepción de riesgo con el consumo de tabaco mencionan que, a pesar de que las mujeres inician a fumar antes que los hombres, ellos fuman con mayor frecuencia ${ }^{19}$ y tienen adecuados niveles de percepción de riesgo de consumo de tabaco. ${ }^{16}$

Lo anterior indica el poco conocimiento que los adolescentes tienen de este fenómeno, lo que se refleja en los altos índices de consumo de estas sustancias. Por lo tanto se planteó el objetivo del presente estudio, el cual fue determinar la relación entre la percepción de riesgo y el consumo de alcohol y tabaco en estudiantes de una preparatoria en Zacatecas.

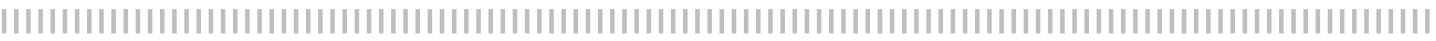

\section{Material y métodos}

El diseño del estudio fue descriptivo, correlacional y comparativo, ${ }^{20}$ la población estuvo conformada por 2,263 sujetos de entre 15 a 21 años de edad, de ambos sexos, estudiantes de una preparatoria del área conurbana de Zacatecas, Zacatecas. El tipo de muestreo que se utilizó fue aleatorio estratificado con asignación proporcional al estrato (hombres y mujeres). La muestra se calculó para una población finita y como parámetro principal a estimar fue una proporción, con un nivel de confianza del 95\% y límite de error de estimación del .05, quedando conformada la muestra final de 326 estudiantes, ${ }^{21}$ se eliminaron dos instrumentos de medición por estar incompletos e ilegibles quedando un total de 324 participantes. Para la selección de los estudiantes de preparatoria se solicitó al Departamento Escolar de la institución educativa el número de grupos que se encontraban registrados de los que se seleccionaron al azar 11 grupos que integraron la muestra final.

Para la recolección de la información se utilizó una Cédula de Datos Personales y de Prevalencia de Consumo de Alcohol y Tabaco (CDPPCAT), que permitió indagar datos sociodemográficos como edad, sexo, estado civil, semestre, así como las prevalencias de consumo de alcohol y tabaco (alguna vez en la vida, en el último año, último mes y en la última semana). Para medir las variables de interés, se aplicaron tres instrumentos: el primero fue el Cuestionario de Percepción de Riesgo Hacia el Consumo de Drogas (CPRCD), ${ }^{19}$ el cual consta de 38 reactivos distribuidos en cinco subescalas: consecuencias negativas del consumo de alcohol, actitud negativa hacia el consumo de drogas lícitas, consumo de drogas lícitas para tener amigos y sensaciones nuevas, consumo de drogas lícitas como afrontamiento y pertenencia, y por último la subescala de consecuencias negativas del consumo de tabaco; con opciones de respuesta de tipo Likert que van desde $1=$ Totalmente en desacuerdo, a $5=$ Totalmente de acuerdo. La escala se convirtió a un índice global con valores de 0 a 100, lo que significa que a mayor puntaje obtenido es mayor la percepción de riesgo para el consumo de este tipo de sustancias. En el presente estudio la escala presentó una consistencia interna $\alpha$ de Cronbach de .909 lo que se considera como un coeficiente aceptable. ${ }^{22}$ 
El segundo instrumento que se aplicó fue el Cuestionario de Identificación de los Trastornos Debidos al Consumo de Alcohol (AUDIT), el cual consta de 10 reactivos de opción múltiple que explora el consumo de alcohol durante el último año. Los resultados del AUDIT fluctúan entre 0 y 40 puntos, a mayor puntaje existe mayor consumo de alcohol. ${ }^{23,24}$ En este estudio el instrumento presentó una consistencia interna $\alpha$ de Cronbach de .811, lo que se considera un coeficiente aceptable. ${ }^{22}$

Otro instrumento que se utilizó fue el Test de tolerancia de Fagerström (CTF) para detectar el consumo de tabaco y estimar el grado de dependencia a la nicotina, el cual consta de 6 reactivos, con una puntuación máxima de 10 puntos, una puntuación mayor de cinco indica dependencia de nicotina..$^{25}$ En el presente estudio el cuestionario de Fagerstrom mostró una consistencia interna menor a .60, lo que se considera suficiente. ${ }^{26}$

El presente estudio se fundamentó en el Reglamento de la Ley General de Salud en materia de Investigación para la Salud. ${ }^{27}$ El cual establece que el desarrollo de la investigación para la salud debe atender aspectos éticos que garanticen la protección de los derechos, el respeto a la dignidad, el bienestar, la participación voluntaria y la confidencialidad de la información de los participantes en el estudio. Además, se obtuvo el consentimiento informado de alguno de los padres, en su caso el asentimiento informado del participante menor de edad, y se contó con el permiso de las autoridades de la institución educativa donde se llevó a cabo el estudio.
Enseguida se visitó a los grupos seleccionados en la fecha y horario establecido por las autoridades de la institución educativa para invitarlos a participar en el estudio, explicarles los objetivos de la investigación, instrumentos de medición y el tiempo requerido para contestarlos. Se hizo hincapié que su participación era voluntaria. A quienes aceptaron participar y que cumplían con el requisito de ser menores de edad, se les entregó los consentimientos y asentimientos informados para contar con el permiso de sus padres o tutores, así como de ellos mismos para participar en el estudio, se concertó la fecha y hora para recabar el consentimiento firmado por el padre o tutor y asentimiento del estudiante; a quienes contaron con el permiso, se les entregaron los instrumentos de medición que contestaron en un tiempo aproximado de 20 minutos, y se resolvieron las dudas que se presentaron. Al finalizar se agradeció a los participantes y a las autoridades de la institución educativa.

Los datos obtenidos fueron procesados en el software SPSS versión 22.0 para Windows. Se utilizó estadística descriptiva (frecuencias, proporciones, medidas de tendencia central y de dispersión) y estadística inferencial, se utilizó la prueba de normalidad de Kolmogorov-Smirnov con corrección de Lilliefors para determinar la normalidad en la distribución de las variables continuas y numéricas, los resultados mostraron que no había normalidad en la distribución de los datos $(p<.05)$, por lo que se decide utilizar la estadística no paramétrica a través de la U de Mann-Whitney y del coeficiente de correlación de Spearman.

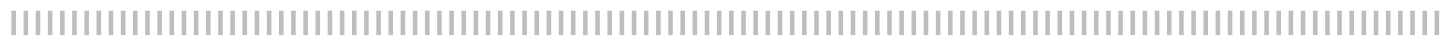

\section{Resultados}

En las características sociodemográficas de los 324 participantes, los resultados muestran predominio del sexo femenino con $54.9 \%$; la edad promedio fue de 16.19 años \pm 1.19 , la gran mayoría eran solteros $(94.4 \%)$, un poco más de la mitad $(59.9 \%)$ se encontraban cursando el segundo semestre de preparatoria. Quienes mencionaron ser usuarios de alcohol, reportan una media de edad de inicio de 14.09 años \pm 2.01 , con edad mínima de 3 
y máxima de 18 años, la cantidad de copas consumidas por ocasión osciló entre 1 hasta 25 y un promedio de $3.16 \pm 2.9$, y más de la mitad (59.0\%) señaló su ingesta 2 a 3 veces por mes, siendo su bebida de preferencia la cerveza; sin embargo, una gran parte presentan un consumo diversificado en un día típico. Con respecto al consumo de tabaco la media de edad de inicio fue de $14.58 \pm 1.82$, edad mínima de 4 y máxima de 18 años, el número de cigarros fumados por ocasión fue de 1 hasta 20 y un promedio de 1.78 , el $40.3 \%$ dijo fumar 2 a 3 veces por mes.

En las prevalencias del consumo de alcohol en los estudiantes de preparatoria, la gran mayoría $(81.8 \%)$ ha consumido alcohol alguna vez en la vida (prevalencia global). Llama la atención que más de una cuarta parte, es decir, $25.9 \%$, continúa haciéndolo en los últimos siete días (prevalencia instantánea) (tabla 1).

\section{Tabla I}

\section{Prevalencia de consumo de alcohol en estudiantes de preparatoria}

\begin{tabular}{|lllllll|}
\hline Consumo de Alcohol & SI & & NO & \multicolumn{3}{c|}{ I.C. $95 \%$} \\
\hline $\mathrm{n}=324$ & $\mathrm{~F}$ & $\%$ & $\mathrm{f}$ & $\%$ & LI & LS \\
\hline Alguna vez en la vida & 265 & 81.8 & 59 & 18.2 & .78 & .86 \\
\hline En el último año & 252 & 77.8 & 72 & 22.2 & .73 & .82 \\
\hline En el último mes & 141 & 43.5 & 183 & 56.5 & .38 & .49 \\
\hline En la última semana & 84 & 25.9 & 240 & 74.1 & .21 & .31 \\
\hline
\end{tabular}

Nota: $f=$ frecuencia,$\%=$ porcentaje, I.C. $95 \%=$ Intervalo de confianza al $95 \%$ para respuestas afirmativas, $L I=$ Limite inferior, $L S=$ Limite superior.

En relación a las prevalencias de consumo de tabaco en estudiantes de preparatoria, un poco menos de la mitad (42.9\%) ha fumado cigarros alguna vez en la vida (prevalencia global), y el $10.8 \%$ lo continúo haciendo en la última semana (prevalencia instantánea) tabla 2.

\section{Tabla 2}

\section{Prevalencia de consumo de tabaco en estudiantes de preparatoria}

\begin{tabular}{|lllllll|}
\hline Consumo de Tabaco & Sí & & NO & \multicolumn{3}{c|}{ I.C. $95 \%$} \\
\hline $\mathrm{N}=324$ & $\mathrm{f}$ & $\%$ & $\mathrm{f}$ & $\%$ & LI & LS \\
\hline Alguna vez en la vida & 139 & 42.9 & 185 & 57.1 & .37 & .48 \\
\hline En el último año & 98 & 30.2 & 226 & 69.8 & .25 & .35 \\
\hline En el último mes & 60 & 18.5 & 264 & 81.5 & .14 & .23 \\
\hline En la última semana & 35 & 10.8 & 289 & 89.2 & .07 & .14 \\
\hline
\end{tabular}

Nota: $f=$ frecuencia,$\%=$ porcentaje, I.C. $95 \%=$ Intervalo de confianza al $95 \%$ para respuestas afirmativas, $L I=$ Limite inferior, $L S=$ Limite superior.

Con respecto al comportamiento del índice del cuestionario de percepción de riesgo en los cuatro tipos de prevalencia de consumo de alcohol a través de la $U$ de Mann-Whitney, en la tabla 3 se observa que no existen diferencias significativas
( $p<.05)$; es decir, los estudiantes que no consumen alcohol presentaron mayores medias de percepción de riesgo en comparación con los estudiantes que sí ingieren bebidas alcohólicas. 


\section{Tabla 3}

Percepción de Riesgo de acuerdo al tipo de prevalencia de consumo de alcohol en estudiantes de preparatoria

\begin{tabular}{|llllll|}
\hline Índice Percepción de Riesgo & $\mathrm{n}$ & $\mathrm{x}$ & $\mathrm{DE}$ & $\mathrm{U}$ & $\mathrm{p}$ \\
\hline Consumo de alcohol último año & & & & 7466.50 & .002 \\
\hline Sí & 252 & 60.06 & 13.59 & & \\
\hline No & 72 & 62.35 & 17.58 & & \\
\hline Consumo de alcohol último mes & & & & 10750.00 & .010 \\
\hline Sí & 141 & 59.11 & 12.96 & & \\
\hline No & 183 & 61.69 & 15.65 & & \\
\hline Consumo de alcohol última semana & & & & & \\
\hline Sí & 84 & 58.01 & 12.55 & & \\
\hline No & 240 & 61.46 & 15.14 & & \\
\hline
\end{tabular}

Notas: $n=324$, = Media,$U$ =U de Mann-Whitney, $p=$ Significancia estadística .

Los resultados del índice del cuestionario de percepción de riesgo en los cuatro tipos de prevalencia de consumo de tabaco a través de la $U$ de Mann-Whitney, muestran que no existen diferencias sig- nificativas ( $p$.05), lo cual significa que los estudiantes que no fuman presentaron mayores medias de percepción de riesgo en comparación con los estudiantes que sí lo hacen (tabla 4).

\section{Tabla 4}

\section{Percepción de Riesgo de acuerdo al tipo de prevalencia de consumo de tabaco en estudiantes de preparatoria}

\begin{tabular}{|llllll|}
\hline Índice Percepción de Riesgo & $\mathrm{n}$ & $\mathrm{X}$ & $\mathrm{DE}$ & $\mathrm{U}$ & $\mathrm{P}$ \\
\hline Consumo de tabaco último año & & & & 8763.00 & .003 \\
\hline Sí & 98 & 57.93 & 12.90 & & \\
\hline No & 226 & 61.71 & 15.13 & & \\
\hline Consumo de tabaco último mes & & & & 5622.50 & .000 \\
\hline Sí & 60 & 56.12 & 11.55 & & \\
\hline No & 264 & 61.58 & 15.02 & & .001 \\
\hline Consumo de tabaco última semana & & & & & 3307.50 \\
\hline Sí & 35 & 54.64 & 11.20 & & \\
\hline No & 289 & 61.28 & 14.79 & & \\
\hline
\end{tabular}

Notas: $n=324$, = Media,$U$ =U de Mann-Whitney, $p=$ Significancia estadística

En la relación entre la percepción de riesgo y el consumo de alcohol se encontró una correlación negativa y significativa entre el índice de AUDIT con el cuestionario de percepción de riesgo (rho $=-.162, p=$ .010), y las subescalas de la actitud negati- va hacia el consumo de drogas lícitas (rho $=-.327^{* *}, p=.000$ ) y el consumo de drogas lícitas para tener amigos y sensaciones nuevas (rho $=-.140^{*}, p=.026$ ). La cantidad de bebidas alcohólicas ingeridas por ocasión y la actitud negativa hacia el con- 
sumo de drogas lícitas (rho $=-.213^{*}, \mathrm{p}=$ .000). Así mismo, se observó esta misma asociación entre el índice de Fagerström y el cuestionario de percepción de riesgo (rho $=-.240^{*}, \mathrm{p}=.017$ ) y las subescalas actitud negativa hacia el consumo de drogas lícitas (rho $=-.259^{*}, p=.010$ ) y el consumo de drogas lícitas para tener amigos y sensaciones nuevas ( $r$ o $=-.237^{*}$, $\mathrm{p}=.019$ ). El número de cigarros fumados por ocasión presentó relación negativa y significativa con el índice de percepción de riesgo ( $r$ o $=-.204^{*}, p=.017$ ); las conse- cuencias negativas del consumo de alcohol ( $r$ o $=-.188, p=.020$ ), la actitud negativa hacia el consumo de drogas lícitas (-.028*, $p=.015)$, y el consumo de drogas lícitas para tener amigos y sensaciones nuevas (rho $=-.174, p=.043$ ), señalan que, $a$ mayor percepción de riesgo, actitud negativa hacia el consumo de drogas lícitas, y percepción de riesgo hacia el consumo de drogas para tener amigos y sensaciones nuevas, es menor el consumo de alcohol y tabaco (tabla 5).

\section{Tabla 5}

Relación entre la percepción de riesgo y el consumo de alcohol y tabaco en estudiantes de preparatoria

\begin{tabular}{|c|c|c|c|c|}
\hline Variable & $\begin{array}{l}\text { Índice } \\
\text { AUDIT } \\
\text { rho }\end{array}$ & $\begin{array}{l}\text { Cantidad de bebidas } \\
\text { alcohólicas ingeridas } \\
\text { en un día típicoc }\end{array}$ & $\begin{array}{l}\text { Índice } \\
\text { Fagerström }{ }^{d}\end{array}$ & $\begin{array}{l}\text { Número de cigarros } \\
\text { fumados en } \\
\text { un día típico }\end{array}$ \\
\hline & $p$ & rho; $p$ & rho; $p$ & rho; $p$ \\
\hline $\begin{array}{l}\text { Índice de Percepción de } \\
\text { Riesgoa }\end{array}$ & $\begin{array}{l}-.162^{*} \\
.010 \\
\end{array}$ & $\begin{array}{l}-.051 \\
.414 \\
\end{array}$ & $\begin{array}{l}-.240^{*} \\
.017 \\
\end{array}$ & $\begin{array}{l}-.204^{*} \\
.017\end{array}$ \\
\hline $\begin{array}{l}\text { Consecuencias negativas } \\
\text { del consumo de alcohol }\end{array}$ & $\begin{array}{l}-.017 \\
.793 \\
\end{array}$ & $\begin{array}{l}.071 \\
.248 \\
\end{array}$ & $\begin{array}{l}-.167 \\
.100 \\
\end{array}$ & $\begin{array}{l}-.188^{*} \\
.028 \\
\end{array}$ \\
\hline $\begin{array}{l}\text { Actitud negativa hacia el } \\
\text { consumo de drogas lícitas }\end{array}$ & $\begin{array}{l}-.327^{* *} \\
.000\end{array}$ & $\begin{array}{l}-.213^{* *} \\
.000\end{array}$ & $\begin{array}{l}-.259^{*} \\
.010 \\
\end{array}$ & $\begin{array}{l}-.208^{*} \\
.015 \\
\end{array}$ \\
\hline $\begin{array}{l}\text { Consumo de drogas lícitas } \\
\text { para tener amigos y } \\
\text { sensaciones nuevas }\end{array}$ & $\begin{array}{l}-.140^{*} \\
.026\end{array}$ & $\begin{array}{l}-.084 \\
.173\end{array}$ & $\begin{array}{l}-.237^{*} \\
.019\end{array}$ & $\begin{array}{l}-.174^{*} \\
.043\end{array}$ \\
\hline $\begin{array}{l}\text { Consumo de drogas lícitas } \\
\text { como afrontamiento y } \\
\text { pertenencia }\end{array}$ & $\begin{array}{l}-.037 \\
.557\end{array}$ & $\begin{array}{l}-.008 \\
.897\end{array}$ & $\begin{array}{l}-.028 \\
.787\end{array}$ & $\begin{array}{l}-.031 \\
.722\end{array}$ \\
\hline $\begin{array}{l}\text { Consecuencias negativas } \\
\text { del consumo de tabaco }\end{array}$ & $\begin{array}{l}-.009 \\
.888\end{array}$ & $\begin{array}{l}-.067 \\
.433\end{array}$ & $\begin{array}{l}-.126 \\
.216\end{array}$ & $\begin{array}{l}-.130 \\
.131\end{array}$ \\
\hline
\end{tabular}

Nota: $p=$ valor de $p ; *=<.05$ (dos colas); $* *=<.01$ (dos colas); rho = Coeficiente de correlación de Spearman; $n^{a}=324 ; n^{b}=252 ; n c=264 ; n^{d}=98 ; n^{e}=98$

\section{| | | | | | | | | | | | | | | | | | | | | | | | | | | | | | | | | | | | | | | | | | | | | | | | | | | | | | | | | | | | | | | | | | | | | | | | | | | | | | | | | | | | | | | | | | | | | | | ||}

\section{Discusión}

En el presente estudio se observa que los estudiantes que participaron en la investigación, inician el consumo de alcohol y tabaco a edades muy tempranas incluso siendo aún niños, debido a que el prome- dio de edad de inicio fue antes de los 15 años (14.09 y 14.58) y como edad mínima a los 3 y 4 años respectivamente; la cantidad de bebidas alcohólicas que consumen por ocasión de 3.16 (1-25), y de cigarros de 1.78 (1-20); la mayoría mencionaron el 
uso de ambas sustancias de 2 a 3 veces por mes, y la bebida que mayormente consumen es la cerveza.

La encuesta nacional de adicciones más reciente (ENCODAT 2017) ${ }^{9}$ y MéndezRuiz y cols ${ }^{17}$ reportan mayor promedio de edad de inicio en el consumo de alcohol (17.9 y 15 años respectivamente). A lo encontrado en este estudio, en tabaco la ENCODAT $2017^{11}$ informa que la edad de inicio es similar a este estudio (14.3 años). Así mismo, estos autores comunican similitud en la cantidad de bebidas alcohólicas consumidas por ocasión (menos de 4 bebidas) $)^{9,17}$ y mayor cantidad de cigarros fumados en un día típico (5.8). ${ }^{11}$

Como se observa en este estudio, la edad de inicio en el consumo de alcohol y tabaco es menor a los estudios mencionados, y lo que es alarmante es el hecho de que se encontraron edades mínimas de 3 y 4 años, lo que se puede explicar por la alta tolerancia en el contexto social, debido a que se considera como parte integral del desarrollo psicosocial y de socialización de una cultura; es decir, esta conducta en los jóvenes puede ser por la influencia social, cultural, familiar y de los compañeros. ${ }^{12}$

En las prevalencias "alguna vez en la vida, en el último año, último mes y última semana de consumo de alcohol y tabaco", los resultados muestran cifras superiores a lo reportado por ENCODAT, 20179,11 y diversos estudios realizados en el ámbito nacional. ${ }^{15,17,18}$ Esto se puede explicar por las características propias del adolescente y también por la falta de supervisión por parte de los profesores debido a la gran cantidad de alumnos que existen en las instituciones públicas de educación media superior. ${ }^{12,-15,17}$

Al comparar el índice de percepción de riesgo entre los cuatro tipos de prevalencias de consumo de alcohol y tabaco a través de la $U$ de Mann-Whitney, se encontró que no existen diferencias significativas ( $p$ $<.05)$; es decir, los estudiantes consumi- dores de alcohol presentaron medias más bajas de percepción de riesgo en comparación con los estudiantes que no ingieren este tipo de sustancias. Uribe y cols $^{19}$ (2011) menciona que la percepción de riesgo relacionada al consumo de drogas está estrechamente vinculada a las creencias sobre las consecuencias negativas; ${ }^{19}$ es decir, cuando los adolescentes perciben mayores consecuencias negativas, es mayor la actitud negativa hacia el consumo de drogas lícitas. ${ }^{17}$

Al relacionar el índice de percepción de riesgo para el consumo de drogas lícitas y el consumo de alcohol y tabaco, se encontraron relaciones negativas y significativas $(p<.05)$ entre el cuestionario de percepción de riesgo y las subescalas consecuencias negativas hacia el consumo de alcohol, la actitud negativa hacia el consumo de drogas lícitas y el consumo de drogas lícitas para tener amigos y sensaciones nuevas, con el índice de AUDIT e índice de Fagerstrom, la cantidad de bebidas alcohólicas y de cigarros consumidos en un día típico, señala que, a mayor percepción de riesgo, es menor el consumo de drogas lícitas. Esto es igual a lo reportado por Méndez-Ruiz y cols $^{17}$ en su estudio con adolescentes en el estado de Nuevo León, reporta correlaciones negativas y significativas entre la percepción de riesgo y el consumo de alcohol.

Uribe y cols ${ }^{19}$ mencionan que el uso frecuente de drogas lícitas (alcohol y tabaco) e ilícitas (marihuana, cocaína, heroína) en adolescentes se relaciona con la percepción del nivel de peligrosidad de las sustancias, así como la actitud hacia su consumo. Al no tener una alta percepción de riesgo se está en mayor peligro de consumir este tipo de sustancias, ya que por un lado se tiene la creencia de que no son tan peligrosas y además la alta permisividad social hacia el consumo, la facilidad para conseguir la droga, y muy probablemente exista la creencia de que pueden tener control si deciden consumir. 


\section{Conclusiones}

Al igual que en México, en el estado de Zacatecas el consumo de alcohol y tabaco representan un problema de salud pública que va en aumento, sobre todo en los adolescentes; como lo reflejan los resultados de este estudio, el cual contribuyó a identificar el incremento de consumo de alcohol y tabaco en estudiantes de una preparatoria en Zacatecas. El consumo se reporta más elevado que en el panorama nacional y la edad en la que inician el consumo de ambas sustancias es más baja. Aunque no se encontraron diferencias significativas entre la percepción de riesgo y los tipos de prevalencias de consumo de alcohol y tabaco, es preocupante el hecho de que más de la cuarta parte de los adolescentes consumieron alcohol en la semana previa al estudio. Sin embargo se encontraron relaciones negativas y con significancia estadística entre la percepción de riesgo para el consumo de drogas lícitas y el consumo de alcohol y tabaco, lo que se interpreta como que a menor percepción de riesgo es mayor el consumo de estas sustancias psicoactivas.

\section{Sugerencias}

Los resultados de este estudio evidencian en los estudiantes de una preparatoria en Zacatecas la presencia de un alto consumo de alcohol y tabaco; sin embargo, el consumo es menor cuando existe alta percepción de riesgo, hallazgo importante para los profesionales del área de la salud, especialmente para enfermería que es clave en fomentar conductas y estilos de vida saludables libres del consumo de sustancias psicoactivas a través de intervenciones dirigidas a reforzar los conocimientos de los daños, efectos y consecuencias del consumo de estas sustancias que permitan en esta población incrementar la percepción de riesgo y fomentar estrategias de afrontamiento ante la influencia de factores de riesgo para el consumo de estas sustancias, por lo que se sugiere:

1. Realizar estudios de investigación con enfoque cualitativo y a través de su discurso se clarifique sus experiencias, expectativas, y lo que significa para ellos el consumo de alcohol y tabaco.

2. Diseñar programas de intervención que contemplen temáticas sobre el efecto del alcohol en el sistema nervioso, el impacto en la salud y social del consumo de alcohol y tabaco; el fortalecimiento de las habilidades para la vida como es el autoestima, la auto-eficacia, la asertividad, el proceso de toma de decisiones adecuadas, como ocupar el tiempo libre; diversión sin asumir altos riesgos; fortalecimiento de valores ante influencias negativas; ejercicios de relajación, animación y reflexión ${ }^{28}$, que fortalezca los conocimientos de los daños, efectos y consecuencias del consumo de drogas lícitas, incrementen la percepción del riesgo, disminuya la edad de inicio.

3. Diseño de un modelo de acompañamiento y reforzamiento de conductas saludables y de autocuidado por parte de profesionales de la enfermería. 


\section{Bibliografía}

1 World Health Organization. Global status report on alcohol and health 2018. Printed in Switzerland. 1-478. Disponible en: https://www.who.int/es/ news-room/detail/21-09-2018-harmful-use-of-alcohol-kills-more-than-3-million-people-each-year-most-of-them-men

2 Organización Panamericana de la Salud. Informe de situación regional sobre el alcohol y la salud de las Américas Washington, DC : OPS. 2015:1-84.

3 Pichon-Riviere A, Bardach A, Augustovski F, Alcaraz $A$, Reynales-Shigematsu $L M$, Teixeira Pinto $M$ et al. Impacto económico del tabaquismo en los sistemas de salud de América Latina: un estudio en siete países y su extrapolación a nivel regional. Rev Panam Salud Pública. 2016; 40(4):213-21.

4 Alcaraz A, Caporale J, Bardach A, Augustovski F, Pichon-Riviere A. Carga de enfermedad atribuible al uso de tabaco en Argentina y potencial impacto del aumento de precio a través de impuestos. Rev Panam Salud Pública. 2016; 40(4):204-12.

5 Bardach A, García Perdomo HA, Ruano Gándara RA, Ciapponi A. Niveles de ingreso y prevalencia de tabaquismo en América Latina: revisión sistemática y metaanálisis. Rev Panam Salud Publica. 2016;40(4):263-71.

6 Vega-López MG., González-Pérez GJ, Flores-Villavicencio, ME, Quintero-Vega PP, Flores-Padilla L, Trejo-Franco J. Tabaquismo en adolescentes: entorno e interacción entre pares. Los casos de Ciudad Juárez y la Zona Metropolitana de Guadalajara. Revista de Educación y Desarrollo, 2017. 43:25-36.

7 Organización Mundial de la Salud. El estado de salud de los adolescentes. 2017.

https://www.who.int/features/factfiles/adolescent health/facts/es/index5.html.

8 Instituto Nacional de Psiquiatría Ramón de la Fuente Muñiz; Instituto Nacional de Salud Pública; Secretaría de Salud. Encuesta Nacional de Adicciones 2011: Reporte de Alcohol Medina-Mora ME, VillatoroVelázquez JA, Fleiz-Bautista C, Téllez-Rojo MM, Mendoza-Alvarado LR, Romero-Martínez M, Gutiérrez-Reyes JP, Castro-Tinoco $M$, Hernández-Ávila M, Tena-Tamayo C, Alvear-Sevilla C, Guisa-Cruz V. México DF, México: INPRFM. 2012. Disponible en: www.inprf.gob.mx, www.conadic.gob.mx, www. cenadic.salud.gob.mx, www.insp.mx.

9 Instituto Nacional de Psiquiatría Ramón de la Fuente Muñiz. Encuesta Nacional de Consumo de Drogas, Alcohol y Tabaco 2016-2017: Reporte de Alcohol Villatoro-Velázquez JA., Resendiz-Escobar, E., Mujica-Salazar, A., Bretón-Cirett, M., Cañas-Martínez, V., Soto-Hernández, I., Fregoso-Ito, D., Fleiz-Bautista, C., Medina-Mora, ME., Gutiérrez-Reyes, J., Franco-Núñez, A., Romero-Martínez, M., y Mendoza-Alvarado, I. Ciudad de México, México. 2017. Disponible en www.inprf.gob.mx; www.conadic. gob.mx; www.cenadic.salud.gob.mx.

10 Instituto Nacional de Psiquiatría Ramón de la Fuente Muñiz; Instituto Nacional de Salud Pública; Secretaría de Salud. Encuesta Nacional de Adicciones 2011:
Reporte de Tabaco Medina-Mora ME, VillatoroVelázquez JA, Fleiz-Bautista C, Téllez-Rojo $M M$, Mendoza-Alvarado LR, Romero-Martínez M, Gutiérrez-Reyes JP, Castro-Tinoco $M$, Hernández-Ávila $M$, Tena-Tamayo C, Alvear-Sevilla C y Guisa-Cruz V. México DF, México: INPRFM. 2012. Disponible en: www.inprf.gob.mx, www.conadic.gob.mx, www. cenadic.salud.gob.mx, www.insp.mx.

11 Instituto Nacional de Psiquiatría Ramón de la Fuente Muñiz. Encuesta Nacional de Consumo de Drogas, Alcohol y Tabaco 2016-2017: Reporte de Tabaco. Reynales- Shigematsu LM. Zavala-Arciniega L, Paz-Ballesteros WC, Gutiérrez-Torres DS, GarcíaBuendía JC, Rodriguez-Andrade MA, Gutiérrez-Reyes, J., Franco-Núñez, A., Romero-Martínez, M. y Mendoza-Alvarado, L. Ciudad de México, México: INPRFM; 2017. Disponible en: www.inprf.gob.mx, www.conadic.gob.mx, www.insp.mx.

12 Ruiz TGM. Medina-Mora IME. La percepción de los adolescentes sobre el consumo de alcohol y su relación con la exposición a la oportunidad y la tentación al consumo de alcohol. Salud Mental. 2014; 37(1):18.

13 Chacón CR. Castro SM. Caracuel CR. Padial RR. Collado FD. Zurita OF. Profiles of alcohol and tobacco use among adolescents from andalusia in the first cycle of secondary education. Health and Addictions. 2016;16(2) 93-104. DOI: 10.21134/haaj. v16i2.266. https://www.researchgate.net/publication/305765914.

14 Ventura-Martínez JL. Castro-Muñoz JR. Carrera-Fernández $\mathrm{P}$. El involucramiento afectivo como factor de protección contra el consumo de sustancias. Horizonte Sanitario. 2017; 16(2):103-109.

15 Mejía MA. Armendáriz GNA. Alonso CMM. Oliva RNN. Calidad de amistad y consumo de alcohol en adolescentes de bachillerato. Revista Cuidarte. 2018;9(3):1-10.

16 Fernández-Castillo E, Molerio PO, Leydiana López TL, Cruz PA, Grau R. Percepción de riesgo respecto al consumo de tabaco en jóvenes universitarios cubanos. Revista Cubana de Medicina General Integral Cuba. 2016; 35(2). http://scielo.

17 Méndez-Ruiz MD, Alonso-Castillo MTJ, AlonsoCastillo MM, Uribe-Alvarado JI. Armendáriz-García NA. Relación de percepción de riesgo y consumo de alcohol en adolescentes. SMAD, Rev. Eletrônica Saúde Mental Álcool Drog. 2015;11(3):161-167. DOI: $\quad$ 10.11606/issn.1806-6976.v11i3p161-167. Disponible en: http://www.redalyc.org/articulo. oa?id $=80342807007$.

18 Méndez-Ruíz MD, Ortiz-Moreno GA, Eligio-Tejada IA, Yáñez-Castillo BG, Villegas-Pantoja MA. Percepción de riesgo y consumo de alcohol en adolescentes mexicanos de preparatoria. Aquichan 2018;18(4): 438-448. DOI: 10.5294/aqui.2018.18.4.6.

19 Uribe AJI., Verdugo, LJC. Y Zacarías SX. (2011) Relación entre percepción de riesgo y consumo de drogas en estudiantes de bachillerato. Psicología y Salud. 21(1): 47-55. Disponible en https://www.uv.mx/ psicysalud/psicysalud-21-1/21-1/J-Isaac-Uribe-Alvarado.pdf. 

Fundamentos para el uso de la evidencia en la práctica de la enfermería. 9a Edición. Filadelfia. Editorial vestigación en Ciencias de la Salud. Monterrey, N. L.

fermería. Desarrollo de la práctica enfermera basada p.1-532. 2016. Babor T. Higgin-Bidle J. Saunders J. Monteiro M. The alcohol use disorders identification test; Guidelines for use in primary care. World Health Organization. 2001.

25 Suárez HL, Dumont HM, de Oliveira NI, Dos Santos VA. Martins G. Análize da utilização do questionário de tolerância de fagerström (QTF) como instrumento de medida da dependência nicotínica. J. Pneumol. 2002.

26 Nunnally, N. C. Psychometric Theory. New York: McGraw-Hill. 1978.

27 Secretaria de Salud Reglamento de la Ley General de Salud en Materia de Investigación para la Salud. México. 2014.

28 Centros de Integración Juvenil, A.C. Programa Armando el Reven. Material didáctico para la prevención de problemas relacionados con el abuso de alcohol y otras drogas. México; 2003. Disponible en: ww.cij.gob.mx. 